\title{
Association of brain-derived neurotrophic factor valine to methionine polymorphism with sexual dysfunction following selective serotonin reuptake inhibitor treatment in female patients with major depressive disorder
}

\begin{abstract}
Introduction: The occurrence of female sexual dysfunction (FSD) in patients with major depressive disorder (MDD) receiving selective serotonin reuptake inhibitors (SSRIs) treatment gives negative impacts on patients' quality of life and causes treatment discontinuation. We aimed to investigate whether genetic polymorphism of identified candidate gene is associated with FSD in our study population.
\end{abstract}

Methods: This is a cross-sectional study. A total of 95 female patients with MDD who met the criteria of the study were recruited and were specifically assessed on the sexual function by trained psychiatrists. Patients' DNA was genotyped for BDNF Val66Met polymorphism using real-time polymerase chain reaction.

Results: The prevalence of FSD in this study is $31.6 \%$. In the FSD group, patients with problematic marriage were significantly more frequent compared with patients who did not have problematic marriage $(\mathrm{P}=0.009)$. Significant association was detected in the lubrication domain with BDNF Val66Met polymorphism ( $\mathrm{P}=0.030)$ using additive genetic model, with even stronger association when using the recessive model $(\mathrm{P}=0.013)$.

Discussion: This study suggested that there was no significant association between BDNF Val66Met with FSD. However, this polymorphism is significantly associated with lubrication disorder in patients treated with SSRIs.

Keyword: Brain-derived neurotrophic factor; Female sexual dysfunction; Major depressive disorder; Pharmacogenetics; Selective serotonin reuptake inhibitor 